\title{
Assessment of voiding dysfunction in Parkinson's disease by the international prostate symptom score
}

Isao Araki, Sadako Kuno

\begin{abstract}
Objectives-To find the incidence of voiding dysfunction in Parkinson's disease and to examine the relation between the voiding dysfunction and various indices of the disease (disease severity, disease duration, age, sex, and treatment with antiparkisonian drugs), the presence of voiding dysfunction was quantitatively estimated in patients sampled on the unselected (consecutive) basis.
\end{abstract}

Methods-Using the international prostate symptom score, lower urinary tract symptoms were quantitatively evaluated in all patients with Parkinson's disease visiting this neurological clinic during 1 month.

Results-Of the 203 patients who had completed the questionnaire, 55 (27\%) were considered to have symptomatic voiding dysfunction. The degree of lower urinary tract symptoms in these patients was well correlated with the severity of the disease rather than with the disease duration or the age. Thirty three (16\%) patients had irritative symptoms alone, whereas three $(1.5 \%)$ patients had obstructive symptoms alone. The irritative and obstructive symptoms were concomitant in $13(6 \%)$ patients. Quality of life was disturbed by lower urinary tract symptoms, and this disturbance paralleled the severity of the disease. The influence of antiparkisonian drugs on the lower urinary tract symptoms was uncertain. The incidence of lower urinary tract symptoms seemed to be independent of sex, but obstructive symptoms were prevalent in male patients.

Conclusions-This study suggests that voiding dysfunction in patients with Parkinson's disease progressively develops at advanced stages ( $\geqslant$ Hoehn and Yahr stage 3 of the disability). The International prostate symptom score is useful in evaluating the voiding dysfunction of neurodegenerative disease in both men and women, not only reflecting prostatic symptoms.

$(\mathcal{F}$ Neurol Neurosurg Psychiatry 2000;68:429-433)

Keywords: Parkinson's disease; voiding dysfunction; urinary symptoms; international prostate symptom score

Voiding dysfunction often occurs in patients with Parkinson's disease and severely disturbs the quality of the patient's life. ${ }^{12}$ The reported incidence of voiding dysfunction in patients with Parkinson's disease ranges from $37 \%$ to $70 \% .^{3-7}$ However, these estimates have been based on the data from patients with Parkinson's disease who visited a urological clinic because of lower urinary tract symptoms (LUTS). In such cases, the samplings of the patients are obviously biased, so that the accurate incidence of voiding dysfunction in such patients remains uncertain. To overcome the sampling defect in these previous studies, we sampled, on an unselected basis, all patients with Parkinson's disease attending our neurological clinic during 1 month. The 203 patients sampled represent about $0.2 \%$ of patients with Parkinson's disease in Japan. The presence of voiding dysfunction was evaluated by the International prostate symptom score (IPSS). The IPSS has been widely used for quantitative evaluation of subjective LUTS (including quality of life) in benign prostatic hyperplasia. ${ }^{8}$ This may be useful in detecting the voiding dysfunction in patients with Parkinson's disease. ${ }^{9-11}$ This evaluation of LUTS makes it possible to quantitatively examine the relation between the incidence of LUTS and the background of the patients such as disease severity, disease duration, age, sex, or treatment with antiparkisonian drugs.

\section{Methods}

QUESTIONNAIRE

During 1 month of July 1997, all patients with Parkinson's disease (208 patients) seen in our neurological clinic had their urinary history taken using the IPSS questionnaire. The questionnaire sheet was handled personally to each patient by a medical or comedical staff member and collected within a few hours. If needed, assistance by medical staff in understanding or completing the questionnaire was permitted, but five out of 208 patients did not complete the questionnaire. The IPSS questionnaire comprises seven questions on LUTS (incomplete emptying, frequent urination within 2 hours, intermittency, urgency, weak stream, straining to start, and nocturia) and an additional question on general satisfaction of urinary conditions. Nocturia was scored from 0 to 5 (five or more times per night) as the actual voiding frequency per night. Other symptoms were scored on a frequency scale from 0 to 5: 0 (not at all); 1 (less than once every five times); 2 (less than half the time); 3 (about half the time); 4 (more than half the time); and 5 (almost always). The scores were aggregated to 


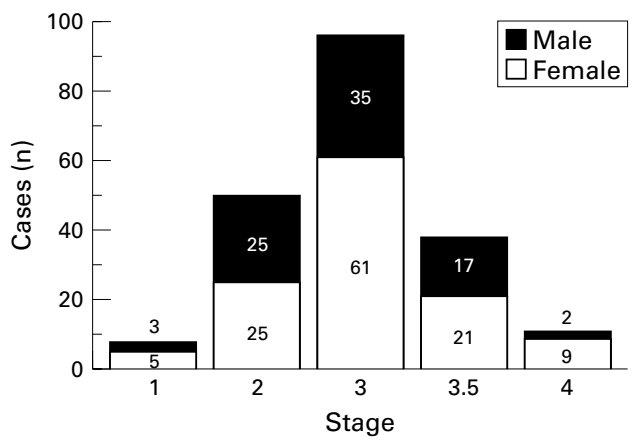

Figure 1 Distribution of male and female patients as a function of modified Hoehn and Yahr stage of disability.

form a symptom index from 0 to 35 . Overall subjective satisfaction on urinary conditions was scored from 0 (delighted) to 6 (terrible) to use as a quality of life (QOL) index.

PATIENTS

Analysis of data was performed in 203 patients who had completed the questionnaire. The sampled patients included 82 men and 121 women, ranging from 37 to 90 (mean 66.6) years old. We examined the distribution of the patients in Hoehn and Yahr stage of disability. ${ }^{12}$ In this classification, the number of patients in stage 3 was found to be disproportionately large $(66 \%)$, and the entire distribution showed a considerable skew in the negative direction. We noticed that the disease state of the patients in stage 3 was not homogenous. To grade the disease stage more finely, we divided the original Hoehn and Yahr stage 3 into stage 3 and stage 3.5. The patients in stage 3 exhibited mild to moderate parkinsonism including some postural instability, and they were physically independent. ${ }^{13}$ However, some of the patients in stage 3 require an attendant person or a wheelchair in the street because of unreliable physical independence, although they can manage daily life without physical care when they are inside the house. We classified these patients as stage 3.5. With this additional stage, the histogram of the patients showed a non-skewed normal distribution as a function of the disease stage (fig 1). The duration of illness ranged from 1 to 37 years with an average of 9.2 years. Most (200/203) patients were receiving various antiparkinsonian drug treatments. These drugs included levodopa (for 193 patients), dopamine receptor agonists (for 153 patients) and anticholinergic agents (for 76 patients).

STATISTICS

All values in the text and tables give the mean (SD). Statistical analysis was made using one way analysis of variance (ANOVA) or linear multiple regression. For analysis of the incidence of symptomatic patients, logistic multiple regression was used. A level of $p<0.05$ was considered to be statistically significant.

\section{Results}

DEPENDENCE ON THE SEVERITY OF PARKINSON'S DISEASE

The symptom index scores significantly increased as the stage of disability advanced
(ANOVA, $\mathrm{p}<0.0001$; table 1 ). At stages 3 or higher of disability, the mean symptom index scores were greater than the mean value reported for control Asian men aged 60 to $69 .{ }^{14}$ Thus voiding dysfunction seems to develop above stage 3 of disability.

Arbitrarily, the patients with a symptom index score of 12 or higher were considered to have symptomatic voiding dysfunction. With this criterion, 55 patients $(27 \%)$ were found to be symptomatic. The incidence of symptomatic patients significantly increased with an increase in the stage of disability, being 33\% and $64 \%$ at stages 3 or higher and stages 4 or higher, respectively (fig 2 ).

IRRITATIVE AND OBSTRUCTIVE URINARY

SYMPTOMS

The IPSS questionnaire includes particular questions about irritative and obstructive urinary symptoms. The frequency, urgency, and nocturia may reflect the state of irritative symptoms (maximal score, 15), whereas incomplete emptying, intermittency, weak stream, and straining at the beginning of urination may depend on obstructive symptoms (maximal score 20$) \cdot{ }^{16}$ Both irritative (ANOVA, $\mathrm{p}<0.0001)$ and obstructive $(\mathrm{p}<0.0005)$ symptom index scores significantly increased with advancing stage of the disability (table 1). When the score in irritative symptoms or obstructive symptoms was $\geqslant 7$ or $\geqslant 9$, the patients were considered to be symptomatic. Among 49 (24.2\%) symptomatic patients, 33 (16.3\%) had irritative symptoms alone, and three $(1.5 \%)$ had obstructive symptoms alone (table 2$)$. Thirteen $(6.4 \%)$ patients manifested both symptoms. Thus, the major urinary symptoms in Parkinson's disease seem to be irritative.

Table 1 The total, irritative and obstructive symptom indices and $Q O L$ index scores at each modified Hoehn and Yahr stage of disability

\begin{tabular}{lrlll}
\hline & \multicolumn{2}{l}{ IPSS } & & \\
\cline { 2 - 4 } \begin{tabular}{l} 
Hohn Yahr stage \\
\cline { 2 - 4 }
\end{tabular} & \multicolumn{1}{l}{ Total } & Irritative & Obstructive & QOL \\
\hline $1(\mathrm{n}=8)$ & $4.3(3.1)$ & $2.4(1.5)$ & $1.9(1.9)$ & $1.1(1.0)$ \\
$2(\mathrm{n}=50)$ & $5.8(4.3)$ & $3.0(2.2)$ & $2.8(2.7)$ & $1.8(1.5)$ \\
$3(\mathrm{n}=96)$ & $8.4(5.9)$ & $4.4(3.1)$ & $4.0(3.7)$ & $2.3(1.6)$ \\
$3.5(\mathrm{n}=38)$ & $11.2(5.1)$ & $6.2(3.2)$ & $5.0(3.5)$ & $3.1(1.6)$ \\
$4(\mathrm{n}=11)$ & $14.2(7.0)$ & $7.5(3.9)$ & $6.7(3.5)$ & $3.7(1.4)$ \\
\hline
\end{tabular}

Each index score is significantly correlated with the stage of disability (one way ANOVA). Values are means (SD).

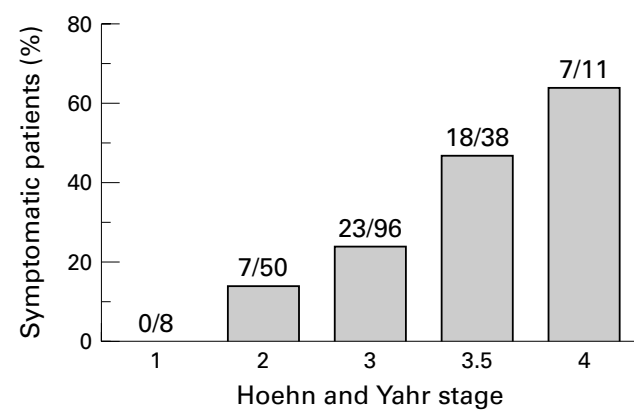

Figure 2 The incidence of patients with symptomatic voiding dysfunction with IPSS score $\geqslant 12$ at different Hoehn and Yahr stages of disability. The incidence is given as \% relative to the total number of patients at each stage. 
Table 2 Incidence of irritative and obstructive symptoms

\begin{tabular}{llll}
\hline $\begin{array}{l}\text { Hoehn and } \\
\text { Yahr stage }\end{array}$ & Irritative alone* & $\begin{array}{l}\text { Obstructive } \\
\text { alonet }\end{array}$ & Both $\neq$ \\
\hline 1 & $0 / 8$ & $0 / 8$ & $0 / 8$ \\
2 & $2 / 50$ & $0 / 50$ & $2 / 50$ \\
3 & $14 / 96$ & $1 / 96$ & $6 / 96$ \\
3.5 & $12 / 38$ & $2 / 38$ & $3 / 38$ \\
4 & $5 / 11$ & $0 / 11$ & $2 / 11$ \\
Total & $33 / 203(16.3 \%)$ & $3 / 203(1.5 \%)$ & $13 / 203(6.4 \%)$
\end{tabular}

* The number of patients with score $\geqslant 7$ in questions related to irritative symptoms alone (maximal score 15).

†The number of patients with score $\geqslant 9$ in questions related to obstructive symptoms alone (maximal score 20).

$\ddagger$ The number of patients with scores $\geqslant 7$ in irritative symptom index and $\geqslant 9$ in obstructive symptom index.

INFLUENCE ON QUALITY OF LIFE

The IPSS questionnaire included a question on general satisfaction of urinary conditions (QOL index). The QOL index scores were significantly correlated with the stage of disability (ANOVA, $\mathrm{p}<0.0001$; table 1 ). When the QOL index score was $\geqslant 4$, the quality of life of the patients was regarded as poor. The incidence of poor QOL increased with disease severity (fig 3). These results suggest that QOL is progressively disturbed as voiding dysfunctions advance. In fact, the QOL index scores were correlated with the symptom index scores $(r=0.74$, $\mathrm{p}<0.0001$ ) (fig 4). Multiple regression analysis $(r=0.75, \mathrm{p}<0.0001)$ indicated that QOL is significantly influenced by the urinary symptoms rather than by the disease severity.

As shown in figures 2 and 3, the incidence of symptomatic patients was parallel with that of the patients with poor QOL. This implies that the symptom index scores $\geqslant 12$ are a reasonable criterion for identifying symptomatic patients.

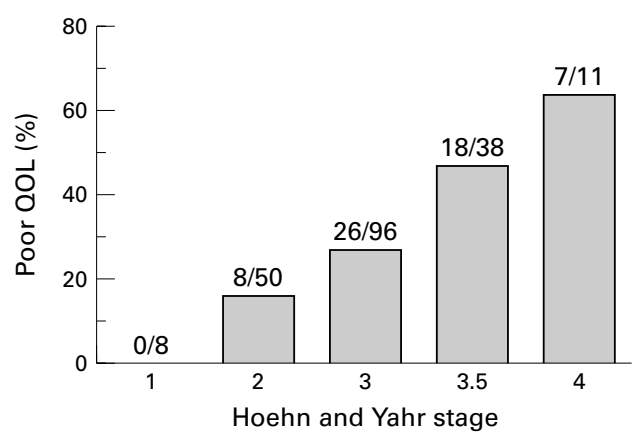

Figure 3 Incidence of patients with poor QOL (QOL index score $\geqslant 4$ ) at each Hoehn and Yahr stage. The incidence is expressed as \% relative to the total number of patients at each stage.

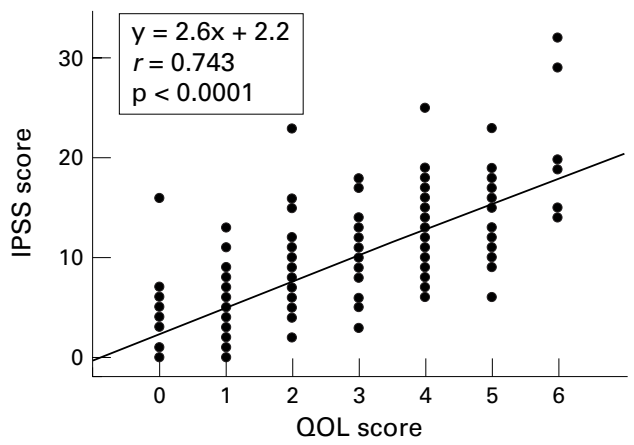

Figure 4 Correlation between QOL index scores and IPSS (symptom index) scores.
DURATION OF ILLNESS AND AGE

We compared the LUTS among three groups of disease duration, $\leqslant 4,5-9$, and $\geqslant 10$ years, or among three groups of age, $\leqslant 59,60-69$, and $\geqslant 70$ years old. There was a significant increase in the mean value of the total symptom index, irritative symptom index, and QOL index scores with the duration of disease (ANOVA, $\mathrm{p}<0.002$, 0.003 , and 0.001 , respectively) or with age $(\mathrm{p}<0.003,0.0001$, and 0.03 , respectively) (data not shown). The obstructive symptom index score was correlated with the duration of disease $(p<0.02)$, but not with age $(\mathrm{p}>0.1)$.

DRUG TREATMENT

Most patients (193/203) had been treated with levodopa. Dopamine receptor agonists were given to 153 patients, and 76 had anticholinergic agents (see methods). We examined the influence of antiparkinsonian drugs on LUTS. There was no indication that the mean value of total, irritative, and obstructive symptom index and QOL index scores differed between patients with and without anticholinergic drugs or between patients with and without dopamine receptor agonists (data not shown). Among 16 patients with significant obstructive symptoms, all had levodopa, all except one had dopamine receptor agonists, and only three had anticholinergic agents. All three patients who had only obstructive symptoms had not been treated with anticholinergic agents.

SEX DIFFERENCE

Infravesical obstructive diseases (for example, benign prostatic hyperplasia), which often show significant LUTS, are prevalent in aged men. The presence of these diseases might have caused the estimate of the incidence of Parkinson's disease related LUTS to be erroneous (an overestimate). To avoid this complication, we also analysed the data only from female patients. In female patients, the total (ANOVA, $\mathrm{p}<0.0005)$, irritative $(\mathrm{p}<0.003)$, and obstructive $(\mathrm{p}<0.006)$ symptom index and QOL index $(\mathrm{p}<0.005)$ scores significantly increased with the disease severity (table 3 ). The symptomatic female patients were 27 of $121(22.3 \%)$, and the incidence increased with the stage of disability (fig 5). Twenty two (18.2\%) patients had irritative symptoms alone, one $(0.8 \%)$ had obstructive symptoms alone, and six (5.0\%) had both symptoms. These results were basically similar to the preceding results from the total population. However, the incidence of obstructive symptoms was higher in male patients $(10.9 \%)$ than in female patients $(5.8 \%)$. In fact, the mean score of obstructive symptoms in male patients (4.8 (SD 3.6), $\mathrm{n}=82$ ) was significantly higher (two tailed $t$ test; $\mathrm{p}<0.005)$ than that in female patients $(3.4$ (SD 3.3), $n=121$ ), whereas the mean scores of total and irritative symptom index and QOL index showed no sex difference.

FURTHER ANALYSIS IN FEMALE PATIENTS

Because of the sex difference in obstructive symptoms, the relation between voiding dysfunction and the indices of the disease was 
Table 3 Total, irritative, and obstructive symptom indices and QOL index scores of female patients at each modified Hoehn and Yahr stage of disability

\begin{tabular}{lrlll}
\hline \multirow{2}{*}{$\begin{array}{l}\text { Hoehn and } \\
\text { Yahr stage }\end{array}$} & \multicolumn{2}{l}{ IPSS } & \multicolumn{1}{l}{} \\
\cline { 2 - 4 } & Total & Irritative & Obstructive & QOL \\
\hline $1(\mathrm{n}=5)$ & $4.8(3.8)$ & $2.6(2.0)$ & $2.2(2.3)$ & $1.2(1.1)$ \\
$2(\mathrm{n}=25)$ & $4.8(3.6)$ & $2.9(2.0)$ & $1.8(2.0)$ & $1.6(1.2)$ \\
$3(\mathrm{n}=61)$ & $8.0(6.6)$ & $4.5(3.3)$ & $3.5(3.6)$ & $2.2(1.6)$ \\
$3.5(\mathrm{n}=21)$ & $9.6(5.5)$ & $5.5(3.6)$ & $4.1(3.6)$ & $2.8(1.6)$ \\
$4(\mathrm{n}=9)$ & $13.1(5.4)$ & $7.2(3.3)$ & $5.9(2.4)$ & $3.4(1.2)$ \\
\hline
\end{tabular}

Each index score is significantly correlated with the stage of disability (one way ANOVA). Values are means (SD).

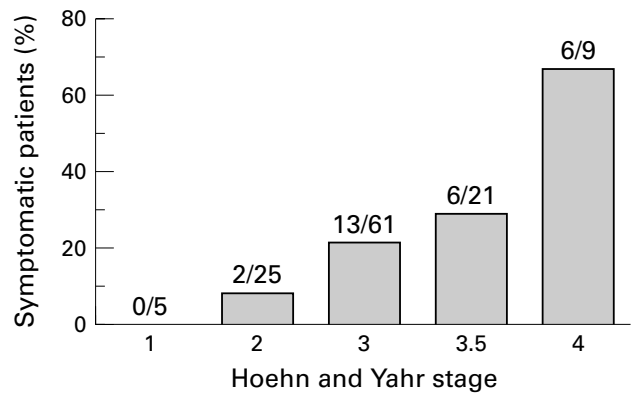

Figure 5 Incidence of female patients with symptomatic voiding dysfunction with IPSS score $\geqslant 12$ at different Hoehn and Yahr stages. The incidence is given by \% relative to the total number of female patients at each stage.

further examined in the data only from female patients.

A single regression analysis indicated that the correlation of the total symptom index score with the disease duration $(r=0.23$, $\mathrm{p}<0.02)$ or the age $(r=0.23, \mathrm{p}<0.02)$ was less significant than that with the stage of disability $(r=0.4, p<0.0001)$. The relation of the total symptom index score with the stage of disability, the disease duration, and the age was examined by the use of linear multiple regression analysis $(r=0.42, \mathrm{p}<0.0002)$. These results suggested that the stage of disability is the better determinant in predicting the total symptom index score (partial regression coefficient $(b)=2.4, \mathrm{p}<0.007)$ than is the disease duration $(b=0.11, \mathrm{p}>0.6)$ or the age $(b=0.075$, $\mathrm{p}>0.08$ ).

The relation between the incidence of symptomatic patients and the three indices of the disease was examined by the use of logistic multiple regression analysis. This analysis again suggested that the stage of disability is the major determinant of the incidence of symptomatic patient $(b=1.3, p<0.02)$, compared with the disease duration $(b=0.02, p>0.7)$ or the age $(b=0.01, \mathrm{p}>0.6)$.

The results of multiple regression analysis in the total population were basically the same as in the female population.

\section{Discussion}

The present study evaluated the incidence of voiding dysfunction in unselected patients with Parkinson's disease attending our neurological clinic. The incidence has been reported to be $37 \%$ to $70 \% .^{3-7}$ In most of these reports, however, the patients were sampled with some bias, and it was unclear what criteria were used to identify symptomatic patients. In our study of 203 unselected patients, the incidence of void- ing dysfunction was $27 \%$ when IPSS scores of 12 or more were regarded as symptomatic. The symptomatic IPSS score used in this study was higher than the score (8 to 19) previously reported for moderate category in the global symptom severity level. ${ }^{16}$ However, it should be noted that the mean IPSS score is higher for Japanese men than for men from western countries. ${ }^{141517}$ Moreover, the incidence of symptomatic patients was almost identical with that of patients complaining of a disturbance of QOL by urinary problems and may be indicative of adequacy of the symptomatic score used in the present study.

The symptom index and QOL index showed a gradual and steady increase with the severity of the disease. The incidence of symptomatic patients also increased with an increase in the severity of disease. The mean value of symptom index scores at stage 3 was greater than that reported for Asian men in the age group $60-69 .^{14}{ }^{15}$ These results suggest that voiding dysfunction develops when severity of disease exceeds stage 3 . One might argue that motor disturbance such as difficulty in reaching the lavatory affects the degree of subjective LUTS and general satisfaction with urinary conditions (QOL). However, individual questions in the IPSS questionnaire on LUTS are posed in terms of the frequency of occurrence of each symptom. Therefore, the influence of motor disturbance on the symptom index score would be minimised. The question on QOL asks the general satisfaction of urinary conditions. In fact, the QOL index score was significantly influenced by the urinary symptoms rather than by the disease severity (disability).

These index scores was much better correlated with the disease severity than with disease duration or age. The influence of age on urinary symptoms in patients with Parkinson's disease was controversial, although it has been reported in normal elderly people that the symptom index scores increase with age ${ }^{141517}$ to the same extent in men and women. ${ }^{910}$ This may reflect the fact that age at onset was different between the patients. As the disease progression may be affected by age at onset, ${ }^{18}$ the disability may not necessarily parallel the age or the disease duration. In fact, the disability was poorly correlated with the age $(r=0.29$, $\mathrm{p}<0.0001$ ), although the relatively high $\mathrm{p}$ value for the disease duration in multiple regression might be caused partly by multicollinearity between the disease severity and duration $(\mathrm{r}=$ $0.51, p<0.0001$ ).

The LUTS can be divided into irritative and obstructive symptoms. Urinary symptoms in Parkinson's disease were found to be mainly irritative, as previously reported..$^{3-7}$ The basal ganglia, including the substantia nigra, have been reported to have inhibitory effects on the micturition reflex. ${ }^{19}$ Therefore, degeneration of dopaminergic neurons of the substantia nigra ${ }^{20}$ might promote irritative symptoms in Parkinson's disease. However, some patients with Parkinson's disease showed obstructive symptoms, in agreement with previous reports. ${ }^{134}$ Because the incidence of obstructive symptoms was higher in male patients than in female 
patients, these symptoms in male patients could be partly attributed to infravesical obstruction (for example, benign prostatic hyperplasia). Levodopa, ${ }^{21}$ anticholinergic agents and dopamine receptor agonists ${ }^{22}$ could affect urinary bladder function. Although the LUTS seemed to be independent of the type of antiparkinsonian drugs, our study cannot tell how a given antiparkisonian drug affects LUTS because most patients examined in this study have received multiple drug treatments. Thus, obstructive symptoms might possibly result from treatment with particular antiparkinsonian drugs. Also, it should be noted that Lewy bodies can be seen in several types of neuron, including central and peripheral components of the autonomic nervous system, in advanced Parkinson's disease. ${ }^{23}$ Thus, obstructive symptoms in patients with Parkinson's disease might result from micturition hyporeflexia due to impairment in the autonomic nervous system. Another possibility is that the patients sampled in our study might have included those with other neurodegenerative diseases (for example, multiple system atrophy) which may manifest hyporeflex bladder dysfunction. At any rate, our study indicates that voiding dysfunction in Parkinson's disease is due largely, if not exclusively, to detrusor hyperreflexia (irritative) in nature.

Although subjective symptoms are not always correlated with urodynamic proof of outflow obstruction, ${ }^{24}{ }^{25}$ it seems clear that the IPSS questionnaire is useful in evaluating voiding dysfunction in neurodegenerative diseases in both men and women. This also implies that the IPSS questionnaire is not specific for evaluating prostatism in men. ${ }^{9-11}$

We thank Drs E Mizuta and S Yamasaki for their kind referral of the patients, $M$ Takechi, A Miyagawa, and $M$ Nakamura for secretarial assistance, and Mr K Kuribayashi for his helpful advice in statistical analysis. This study was supported in part by advice in statistical analysis. This study was supported in part by Ministry of Health and Welfare.

1 Porter RW, Bohrs E, Hymann W. Distortion of extrapyramidal-visceral interrelationships in Parkinson's syndrome. In: Gillingham FJ, Donaldson IML, eds. Third syndrome. In: Gillingham Fo, Donaldson IML, eds. Third Symposium

2 Olanow CW, Koller WC. An algorithm (decision tree) for the management of Parkinson's disease: treatment guidelines. Neurology 1998;50(suppl 3):1-57.

3 Andersen JT, Bradley WE. Cystometric, sphincter and electromyographic abnormalities in Parkinson's disease. 7 Urol 1976;116:75-8

4 Andersen JT. Disturbances of bladder and urethral function in Parkinson's disease. Int Urol Nephrol 1985;17:35-41.
5 Berger Y, Salinas JN, Blaivas JG. Urodynamic differentiation of Parkinson disease and the Shy-Drager syndrome. Neurourol Urodyn 1990;9:117-21.

6 Hattori T, Yasuda K, Kita K, et al. Voiding dysfunction in Parkinson's disease. Fapanese fournal of Psychiatry and Neurology 1992;46:181-6.

7 Stocchi F, Carbone A, Inghilleri M, et al. Urodynamic and neurophysiological evaluation in Parkinson's disease and multiple system atrophy. $\mathcal{f}$ Neurol Neurosurg Psychiatry 1997;62:507-511.

8 Cockett ATK, Aso Y, Denis L, et al. Recommendations of the International Consensus Committee concerning: 1 . Prostate symptom score (I-PSS) and quality of life assessment, 2. Diagnostic work-up of patients presenting with symptoms suggestive of prostatism, 3. Patients evaluation for research studies, and 4. BPH treatment. In: Cockett International Consultation on Benign Prostatic Hyperplasia. Paris: Scientific Communication, 1991:279-340.

9 Lepor H, Machi G. Comparison of AUA symptom index in unselected males and females between 55 and 79 years of age. Urology 1993;42:36-41.

10 Chai TC, Belville WD, McGuire EJ, et al. Specificity of the American Urological Association symptom index: comparison of unselected and selected samples of both sexes. $\mathcal{F}$ Urol 1993;150:1710-13.

11 Chancellor MB, Rivas DA, Keeley FX, et al. Similarity of the American Urological Association symptom index among men with benign prostate hyperplasia (BPH), urethral men with benign prostate hyperplasia (BPH), urethral without outlet obstruction. Br f Urol 1994;74:200-3.

12 Hoehn MM, Yahr MD. Parkinsonism: onset, progression, and mortality. Neurology 1967;113:427-42.

13 Lang AE, Fahn S. Assessment of Parkinson's disease. In: Munsat TL, ed. Quantification of neurological deficit Stoneham: Butterworths, 1989;18:613-20.

14 Tsukamoto T, Kumamoto Y, Masumori N, et al. Prevalence of prostatism in Japanese men in a community-based study with comparison to a similar American study. $\mathcal{F}$ Urol 1995; 154:391-5.

15 Homma Y, Kawabe K, Tsukamoto T, et al. Epidemiologic survey of lower urinary tract symptoms in Asia and Australia using the international prostate symptom score. Int $\mathcal{F}$ Urol 1997;4:40-6.

16 Barry MJ, Fowler FJ Jr, O'Leary MP, et al. The American Urological Association symptom index for benign prostatic hyperplasia. The Measurement Committee of the American Urological Association. F Urol 1992;148:1549-57.

17 Sagnier PP, Girman CJ, Garraway M, et al. International comparison of the community prevalence of symptoms of prostatism in four countries. Eur Urol 1996;29:15-20.

18 Diamond SG, Markham CH, Hoehn MM, et al. Effect of age at onset on progression and mortality in Parkinson's disease. Neurology 1989;39:1187-90.

19 Lewin RJ, Dillard GV, Porter RW. Extrapyramidal inhibition of the urinary bladder. Brain Res 1967;4:301-7.

20 Alvord EC Jr, Forno LS, Kusske JA, et al. The pathology of parkinsonism: a comparison of degenerations in cerebral parkinsonism: a comparison of degenerations in

21 Benson GS, Raezer DM, Anderson JR, et al. Effect of levodopa on urinary bladder. Urology 1976;7:24-8.

22 Yoshimura N, Mizuta E, Kuno S, et al. The dopamine D1 receptor agonist SKF 38393 suppresses detrusor hyperreflexia in the monkey with parkinsonism induced by 1-methyl-4-phenyl-1,2,3,6-tetrahydropyridine (MPTP). Neuropharmacology 1993;32:315-21.

23 Bethlem J, Den Hartog Jager WA. The incidence and characteristics of Lewy bodies in idiopathic paralysis agitans (Parkinson's disease). I Neurol Neurosurg Psychiatry 1960; 23:74-80.

24 Jensen KM, Bruskewitz RC, Iversen P, et al. Spontaneous uroflowmetry in prostatism. Urology 1984;24:103-9.

25 Schafer W, Rubin H, Noppeney R, et al. Obstructed and non-obstructed prostatic obstruction: a plea for urodynamic objectivation of bladder outflow obstruction in benign prostatic hyperplasia. World f Urol 1989;6:198-203. 